\title{
Non-retinotopic feature integration decreases response-locked brain activity as revealed by electrical neuroimaging
}

\author{
Gijs Plomp ${ }^{\mathrm{a}, *}$, Manuel R. Mercier ${ }^{\mathrm{b}}$, Thomas U. Otto ${ }^{\mathrm{a}, \mathrm{c}}$, Olaf Blanke ${ }^{\mathrm{b}}$, Michael H. Herzog ${ }^{\mathrm{a}}$ \\ a Laboratory of Psychophysics, Brain Mind Institute, EPFL, Lausanne, Switzerland \\ b Laboratory of Cognitive Neuroscience, Brain Mind Institute, EPFL, Lausanne, Switzerland \\ ' Laboratoire Psychologie de la Perception, Université Paris Descartes, Paris, France
}

\section{A R T I C L E I N F O}

\section{Article history:}

Received 8 May 2009

Revised 8 June 2009

Accepted 9 June 2009

Available online 21 June 2009

\begin{abstract}
A B S T R A C T
When presented with dynamic scenes, the brain integrates visual elements across space and time. Such nonretinotopic processing has been intensively studied from a psychophysical point of view, but little is known about the underlying neural processes. Here we used high-density EEG to reveal neural correlates of nonretinotopic feature integration. In an offset-discrimination task we presented sequences of lines for which feature integration depended on a small, endogenous shift of attention. Attention effects were observed in the stimulus-locked evoked potentials but non-retinotopic feature integration was reflected in voltage topographies time-locked to the behavioral response, lasting for about $400 \mathrm{~ms}$. Statistical parametric mapping of estimated current densities revealed that this integration reduced electrical activity in an extensive network of brain areas, with the effects progressing from high-level visual, via frontal, to central ones. The results suggest that endogenously timed neural processes, rather than bottom-up ones, underlie non-retinotopic feature integration.
\end{abstract}

(C) 2009 Elsevier Inc. All rights reserved.

\section{Introduction}

Visual processing is often implicitly or explicitly assumed to be retinotopic. On the retina, neighboring elements of the external world project onto neighboring photo-receptors, creating a two-dimensional map of the visual field. This retinotopic coding principle is preserved in much of the visual cortex, including the primary visual areas (Engel et al., 1994; Tootell et al., 1998), the lateral occipital cortex (Gardner et al., 2008; Tootell et al., 1995), and parts of the parietal cortex (Sereno et al., 2001). Despite this predominant retinotopic organization, it has been shown that even very basic visual processing can be non-retinotopic. For example, when a line stimulus is quickly followed by a grating, the lines in the grating inherit the features of the preceding line, such as its orientation, its motion, or its offset (Herzog and Koch, 2001). Thus, features presented at one retinotopic location are attributed to another one. These non-retinotopic attributions are not errors of the visual system but rather reflect systematic visual processing that maintains the identity of perceptual objects across space and time (Otto et al., 2009) and follows the rules of perceptual grouping (Aydin et al., 2008, 2009; Öğmen et al., 2006; Otto et al., 2009). Similar non-retinotopic effects have been demonstrated for luminance (Shimozaki et al., 1999), color (Nishida et al., 2007; Watanabe and Nishida, 2007), shape (Öğmen et al., 2006; Otto et al., 2006), size (Kawabe, 2008), and the conjunction

\footnotetext{
* Corresponding author. EPFL SV BMI LPSY, Station 19, CH-1015, Lausanne, Switzerland. Fax: +41216931749.

E-mail address: gijs.plomp@epfl.ch (G. Plomp).
}

of features (Cavanagh et al., 2008). Although non-retinotopic processing is relatively well described from a psychophysical point of view, little is known about how it operates on a neural level. Here we investigated non-retinotopic processing using high-density EEG techniques.

Non-retinotopic processes can be reliably evoked using the sequential metacontrast paradigm (see Fig. 1 and Supplementary Movie 1 for an animation; Otto et al., 2006). In sequential metacontrast, a central line is quickly followed by a sequence of flanking lines, creating the impression of two motion streams moving in opposite directions. The flanking lines render the central line invisible, as in classical metacontrast masking (Bachman, 1994; Breitmeyer and Öğmen, 2006). In spite of this invisibility, the features of the central line can, surprisingly, be perceived at the flanks. For example, when the central line has a small Vernier offset to the left, the flanking lines in the motion stream look offset to the left, even though they are presented without offset. The features of the central line are not only mislocalized, they also integrate with features of the flanking lines. When one of the flanks has an offset opposite to that of the central line, the two offsets cancel each other out through non-retinotopic feature integration. In this process, the initially presented feature (the central offset) integrates with the subsequently presented feature (the flank offset).

Non-retinotopic feature integration depends strongly on attention (Otto et al, 2006). This allowed us to contrast integration and nonintegration in physically identical stimuli, using a fully crossed factorial design. We asked observers to indicate the perceived offset direction in one of the two motion streams. Only when observers attend to the stream that contains the flank offset (see Fig. 1), non- 

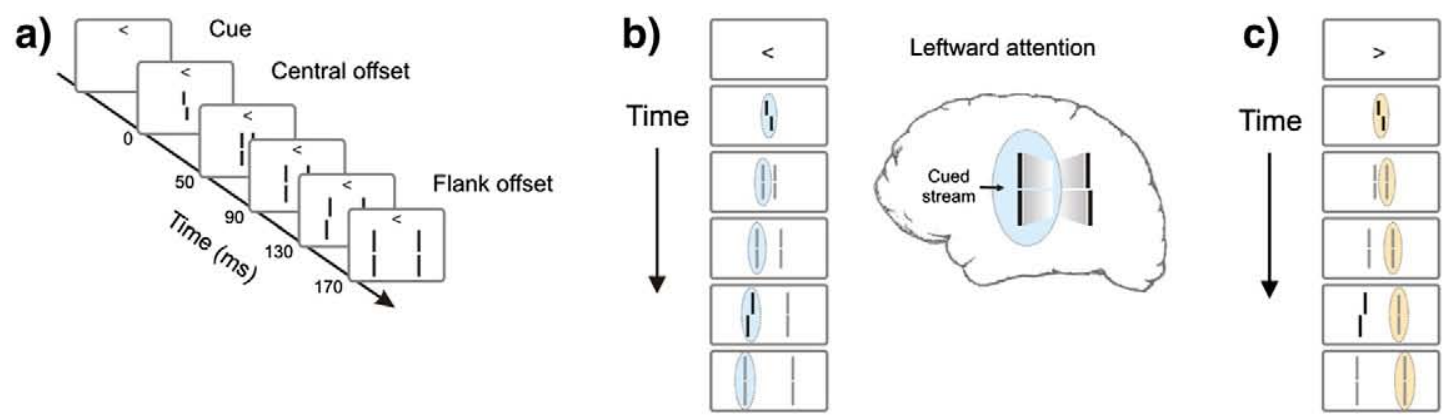

Rightward attention

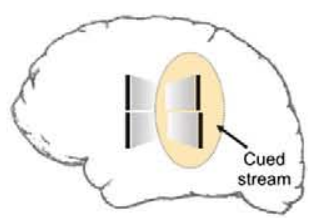

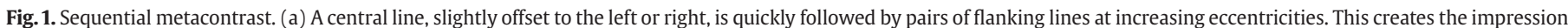

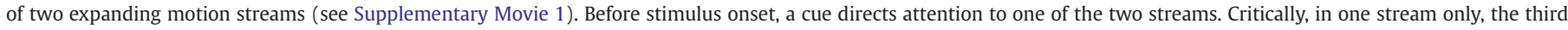

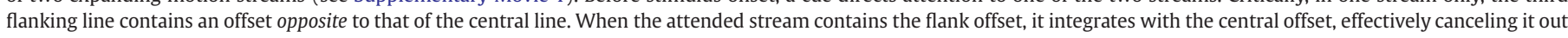

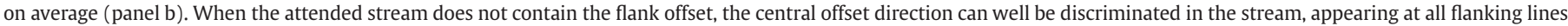
even though the flanks had no offset. This is illustrated in panel c). We presented all possible combinations of attended stream and flank offset stream to the observers.

retinotopic feature integration occurs. This way, a slight, endogenous, shift of attention determines whether or not the two features integrate. This attention-dependence occurs even though the nonattended flank offset falls within the foveated region, suggesting that attention substantially modifies how each stream is processed. Accordingly, we expected an evoked effect of attended stream in early visual processing, similar to that of selective attention to extrafoveal stimuli (Heinze et al., 1990; Hillyard and Anllo-Vento, 1998; Kastner et al., 1999).

Our main interest was to compare feature integration with nonintegration in the evoked potentials (EPs) and their estimated underlying electrical brain sources. At each moment in time, the sum of electric brain activity forms a spatially distributed pattern of electric potentials on the scalp. The topography of these potentials typically alternates between quasi-stable states, a pattern that can be readily observed in the spontaneous EEG (Lehmann, 1984; Lehmann et al. 1998) as well as in averaged EPs (Lehmann and Skrandies, 1980; Blanke et al., 2005). Changes in the topography of scalp potentials reflect large-scale changes in brain activity. Reversely, when the scalp topography is stable, it can be assumed that the underlying brain activity is stable as well. This because a single electrical brain state cannot project in more than one way to the scalp. Stable topographies therefore can be taken to reflect specific brain states. These states have been called functional microstates (Lehmann et al., 1987; PascualMarqui et al., 1995; Michel et al., 2001, 2004) because they reflect distinct patterns of brain activity and computational processes. We identified microstates in the EP data using a topographical clustering algorithm (Lehmann et al., 1987; Pascual-Marqui et al., 1995; Michel et al., 2001, 2004; Murray et al., 2005, 2008; Blanke et al., 2005; Arzy et al., 2006; Thierry et al., 2007). We used the results of this topographic analysis to identify microstate differences between, and commonalities across the experimental conditions. We then estimated the underlying electrical brain activity at the relevant latencies using a Local Auto-Regressive Averages (LAURA, Grave de Peralta Menendez et al., 2004) inverse solution and applied statistical parametric mapping (SPM; Friston et al., 1995; Worsley et al., 1992) on a timepoint by time-point basis to identify the latencies and brain regions where activity reflected non-retinotopic feature integration.

We expected that EP differences between integration and nonintegration would be time-locked to stimulus onset, occurring at latencies of higher-level object processing, around 100 and $200 \mathrm{~ms}$ after presentation of the flank offset. We furthermore expected the underlying source differences to be localized in areas of visual cortex with a largely non-retinotopic organization. Because non-retinotopic processes essentially maintain object identity across space and time (Otto et al., 2009), we expected to find integration effects in higherlevel visual areas that code for objects (i.e. grouped features), such as the lateral occipital complex (LOC; Grill-Spector et al., 2001) or the Fusiform Gyrus (FG). In these areas, we expected decreased activity when the two offsets integrated, because the central offset effectively cancels out.

Because motion is critical to non-retinotopic feature integration (Breitmeyer et al., 2008; Otto et al., 2009), we expected additional activity differences in the Middle Temporal (MT) area. This area codes for basic and complex properties of motion (Born and Bradley, 2005) and may well reflect non-retinotopic integration effects. Here too, we expected decreased activity when the two offsets integrated.

The results showed very strong stimulus-locked effects of attention. The integration effects, however, were time-locked to the behavioral response, extending from around 650 to $250 \mathrm{~ms}$ before observers pressed a button to indicate the perceived offset direction. At these latencies, feature integration was reflected in an extended network of areas, decreasing activity in high-level visual areas, including FG and MT, and subsequently in frontal and central ones.

\section{Materials and methods}

\section{Observers}

Twelve observers ( 3 female) took part, with ages ranging between 30 and 22 (mean 26). They had normal or corrected-to-normal vision with visual acuity of 1.0 in at least one eye (Bach, 1996). Observers were right-handed, as assessed by the Edinburgh Inventory (Oldfield, 1971). All observers were paid for their participation, except for the two participating authors (TO and GP). All procedures were in accordance with the Declaration of Helsinki and approved by the local ethics committee. Observers gave informed consent before the experiment.

\section{Stimuli and apparatus}

The stimuli appeared on an X-Y-display (HP-1332A) controlled by a PC via fast 16 bit D/A converters. Stimuli were composed of dots drawn with a dot pitch of $300 \mu \mathrm{m}$ at a dot rate of $1 \mathrm{MHz}$. The dot pitch was selected so that the dots slightly overlapped (i.e. the dot pitch was of the same magnitude as the dot size or line width). The refresh rate was $200 \mathrm{~Hz}$. Stimulus luminance was $80 \mathrm{~cd} / \mathrm{m}^{2}$, as measured with a Minolta LS-100 luminance meter by means of a dot grid. Recordings were done in an electrically shielded room with the observers seated at 1.5 meter from the monitor. The room was dimly lit (approximately $0.5 \mathrm{~lx}$ ), the background luminance on the screen was below $1 \mathrm{~cd} / \mathrm{m}^{2}$.

We used a sequential meta-contrast paradigm (Otto et al., 2006) with one central line followed by four pairs of flanking lines (Fig. 1). The central line consisted of two segments of $10^{\prime}$ (arc min) length separated by a vertical gap of $1^{\prime}$. The length of the first pair of flanks was $11.6^{\prime}$ and increased progressively by $1.6^{\prime}$ for the following flanking lines. The horizontal distance from the center increased progressively by $3.2^{\prime}$ with each flank. All lines were presented for $20 \mathrm{~ms}$. The 
stimulus onset asynchrony (SOA) between the central line and the first pair of flanking lines was $50 \mathrm{~ms}$, and $40 \mathrm{~ms}$ between subsequent flanking lines. The entire stimulus lasted $190 \mathrm{~ms}$.

The lower segment of the initial central line was slightly offset to the left or right. This offset (mean $1.72^{\prime}$, s.d. $0.4^{\prime}$ ) was fixed such that individual discrimination accuracy was around $75 \%$. Of the third pair of flanking lines, one contained no flank offset and the other was offset in the direction opposite to the central offset. The flank offset size (mean $0.83^{\prime}$, s.d. $0.22^{\prime}$ ) was individually calibrated such that performance was at chance when the flank offset was at the attended stream. The central and flank offset values were determined on a day preceding the EEG recordings using an adaptive method (PEST; Taylor and Creelman, 1967).

We used a fully crossed three-way factorial design of the factors Central Offset Direction (left, right), Attended Stream (left, right) and Flank Offset Stream (left, right stream). In this design non-retinotopic feature integration is operationalized as the interaction effect between Attended Stream and Flank Offset Stream. We presented all conditions randomly interleaved and equally often within a run. A run lasted approximately $4 \mathrm{~min}$ and consisted of 80 trials, i.e. 10 repetitions of each condition. For each observer 10 runs were recorded so that each of the eight conditions was repeated 100 times in total.

We used the sequential metacontrast paradigm because it is resilient against cognitive strategies (Otto et al., 2006). Cognitive strategies are unlikely to affect the results, even in experienced observers, because the central line itself stays invisible, due to metacontrast making, and the central offset and flank offset are not perceived as separate events.

\section{Procedure}

A trial started with a valid location cue, either " $<$ " or ">”, that directed attention to the left or right stream. The cue was presented about half a degree of visual angle above the central line and stayed on screen until the response. Observers were instructed to fixate the cue during the entire trial. After cue onset, the stimulus appeared at a random interval between 1250 and $1550 \mathrm{~ms}$. The observer's task was to attend to the cued stream and determine whether the perceived offset was to the left or right. We instructed observers to emphasize accuracy over speed and to refrain from moving and blinking during the trails. Responses were given by pressing one of two hand-held buttons with the left or right thumb. When the response took longer than $3000 \mathrm{~ms}$, the trial was aborted and repeated later on in the run. Between runs small breaks were offered.

\section{EEG recording and data processing}

The EEG was recorded with a Biosemi Active Two system (Biosemi, Amsterdam, the Netherlands). We recorded with $192 \mathrm{Ag}-\mathrm{AgCl}$ sintered active electrodes positioned in a cap with good coverage of the entire scalp. The cap placement was adjusted individually such that the $\mathrm{Cz}$ electrode was located halfway between inion and nasion, and equally far from each ear. The recording was referenced to the CMS-DRL ground, a feedback loop that keeps the montage potential close to amplifier zero. We recorded the electro-oculogram (EOG) with electrodes $1 \mathrm{~cm}$ above and below the dominant eye, and $1 \mathrm{~cm}$ lateral to the left and right outer canthus. The recording sampling rate was $2048 \mathrm{~Hz}$. Off-line, the data were down sampled to $512 \mathrm{~Hz}$ and band-pass filtered between 1 and $40 \mathrm{~Hz}$ using a Butterworth filter with $-12 \mathrm{db} /$ octave roll-off. We visually inspected all data to determine noisy electrodes. Trials without a response were not further processed.

We calculated both stimulus-locked and response-locked EPs. For the stimulus-locked analysis, we selected epochs between -100 and $600 \mathrm{~ms}$ around stimulus onset. We ran semi-automatic artifact detection with a threshold of $75 \mu \mathrm{V}$ in both EEG and EOG channels and removed epochs that contained artifacts. Across observers, $4 \%$ of epochs were rejected on average. For the response-locked analysis we selected epochs from $-700 \mathrm{~ms}$ to the button press and did artifact rejection in the same way as for the stimulus-locked analysis. We removed all epochs that were excluded from that analysis plus epochs that showed artifacts up to the button press. For the response-locked analysis, on average $17 \%$ of epochs were rejected.

We averaged the selected epochs within observer and condition, resulting in 8 averages per observer, per analysis. DC drift was removed by subtracting the average across the epoch from each amplitude. No baseline correction was applied. We then interpolated (Perrin et al., 1987) the noisy channels in individual recordings. The mean number of interpolated channels was 3 (s.d.=2) across observers. Grand-averages per condition were computed using the average reference after normalizing individual averages to the global field power (GFP) to assure even contributions of observers to the grand-average amplitudes. The GFP is the standard deviation of the electrical potentials across the scalp and is a spatial measure of the response strength (Lehmann and Skrandies, 1980).

\section{Topographic pattern analysis}

The EP analysis was based on the examination of the spatial variations of the scalp voltage topographies over time and between conditions (Michel et al., 2001, 2004; Blanke et al., 2005; Murray et al., 2005). To examine topographies we segmented the continuous grandaverage EEG epochs into a succession of discrete Evoked Potential maps (EP maps). EP maps are temporarily stable scalp topographies that reflect microstates of the brain. We identified EP maps from the grand-averaged data using the Atomize and Agglomerate Hierarchical Clustering (AAHC) algorithm (Murray et al., 2008, see also http:// brainmapping.unige.ch/Cartool.htm), a spatio-temporal clustering algorithm (Lehmann et al., 1987; Pascual-Marqui et al., 1995; Michel et al., 2001,2004; Murray et al., 2005; Blanke et al., 2005; Arzy et al., 2006; Thierry et al., 2007). This topographical analysis summarizes each epoch by a limited number of EP maps. It segments epochs into successions of EP maps without prior assumptions about the temporal characteristics of the data. This is possible because scalp topographies do not randomly change over time but remain stable in a certain configuration for certain periods and then change to a new stable spatial configuration.

The number of EP maps that best explains the whole data set was determined by the absolute minimum in the Cross Validation (CV) value (Pascual-Marqui et al., 1995). The CV value is a ratio of the global explained variance of the AAHC result and the degrees of freedom in the model (the number of maps). Therefore, the number of EP maps is determined in a data-driven way, optimizing the fit to the epochs while keeping the number of maps as low as possible.

The EP maps of the group-averaged data were inspected for commonalities across conditions and for differences between them. Differences in EP maps point to differences in the underlying brain activity because whenever the spatial configuration of the electric field on the scalp changes, the underlying electrical sources must have changed as well (Arzy et al., 2006; Blanke et al., 2005; Michel et al., 2004). To validate the grand-average topographical differences, we fitted the EP maps to the individual data using spatial fitting procedures. For each time-point of the individual epochs, the instantaneous scalp topography was compared to each EP map by means of normalized spatial correlation. Each time-point was then labeled according to the EP map with which it correlated highest. From this back-fitting procedure we determined the duration that a given EP map was observed for a given condition across observers (frequency of occurrence). For each effect tested across observers, the time period for back-fitting was defined on the basis of the grand-average topographical analysis and all EP maps across conditions were used. Three-way repeated measures ANOVAs 
with EP map as within-subject factor were calculated to assess systematic changes in EP map duration. The EEG data processing and topographical analyses were performed using the Cartool software by Denis Brunet (http://brainmapping.unige.ch/Cartool. htm).

\section{Source analyses}

To estimate current densities for each condition and observer we used a Local Auto-Regressive Average (LAURA) inverse solution (Grave de Peralta Menendez et al., 2004). LAURA is a linear, distributed inverse solution that is based on biophysical constraints, producing adequate source localizations with high temporal resolution (Arzy et al., 2006; Blanke et al., 2005; Mercier et al., 2009). Within the gray matter of the Montreal Neurological Institute's (MNI) 152 template brain, we defined a solution space of 4022 evenly spread source points. We transformed the MNI volume to a best fitting sphere (SMAC model; Spinelli et al., 2000), and used a 3-shell spherical head model to calculate the lead field for the 192 electrodes and the LAURA inverse solution. This way, we estimated current densities throughout the source space for each participant at each time-point for each of the eight conditions.

We then applied statistical parametric mapping (SPM; Friston et al., 1995; Worsley et al., 1992) on the current density amplitudes throughout the source space, using three-way repeated measures ANOVAs (Pinheiro and Bates, 2000) with the factors of Attended Stream (left, right), Flank Offset Stream (left, right stream) and Central Offset Direction (left, right) and observers as a random factor. We restricted the SPM analyses to latencies where the topographic analysis showed systematic differences between conditions. We controlled for multiple testing by setting the thresholds for statistical significance such that the false discovery rate (Benjamini and Hochberg, 1995; Genovese et al., 2002) was 5\% for each effect, at every time-point. These analyses were done in $\mathrm{R}$ (R-Development-Core-Team, 2008). We report locations of significant difference in Talairach coordinates (Talairach and Tournoux, 1988).

For each of the brain regions showing significant non-retinotopic integration effects, we correlated the individual current densities at the latency and source point of minimal $p$-value with the behavioral data. Accuracy was defined as the proportion of responses in accord with the central offset direction. We linearly regressed individual Zscores of accuracy in the relevant conditions and calculated $R^{2}$ of the significant (all observed $p<0.01$ ) correlations.

\section{Results}

\section{Behavioral analysis}

We analyzed RT and accuracy as a function of Attended Stream (left, right stream), Flank Offset Stream (left, right stream) and Central Offset Direction (left, right offset) in a repeated measures ANOVA (Pinheiro and Bates, 2000) with observers as random factor. The accuracy analysis showed an interaction between Attended Stream and Flank Offset Stream, $F_{(1,77)}=73.92, p<0.001$. As expected, the two offsets integrated when the flank offset was in the attended stream, but not when it was in the unattended stream (Fig. 2). This interaction effect was also observed for RT (mean $=886 \mathrm{~ms}$, s.d. $=302 \mathrm{~ms}$ ), $F_{(1,77)}=5.43, p<0.05$. Responses were quicker when the flank offset was in the non-attended stream. In addition, an interaction between Attended Stream and Vernier Offset Direction was observed, both in RT and accuracy, which we describe in full in Supplementary Figure 1.

\section{Stimulus-locked analysis}

Topographical analysis of the grand-average stimulus-locked EPs revealed differences between conditions from around $100 \mathrm{~ms}$ after stimulus onset, see Fig. 3a. At three latencies, differences for Attended Stream were observed. First, between 106 and $158 \mathrm{~ms}$, EP map 3 was more expressed for leftward attention and the combination of map 4 and 5 more for rightward attention, $F_{(1,11)}$ $=67.35, p<0.001$. This effect is illustrated in Fig. 3b which shows the traces of the left and right occipital electrode with the maximum evoked amplitude for rightward and leftward attention respectively. Second, between 158 and $182 \mathrm{~ms}$, EP map 7 was more expressed for attention to the left than map 8, $F_{(1,11)}=34.66$, $p<0.001$. Third, between 262 and $372 \mathrm{~ms}$ map 12 was more expressed for attention to the left and map 11 more for attention to the right, $F_{(1,11)}=14.10, p<0.005$. In addition, we obtained an interaction between Attended Stream and Vernier Offset Direction (see Supplementary Figure 1). No other significant effects were observed.

The topographical analysis revealed extensive attention effects between around 100 and $180 \mathrm{~ms}$, and between around 250 and $370 \mathrm{~ms}$ after stimulus onset. The early attention effect constitutes a classical P1 attention effect (Heinze et al., 1990; Hillyard and AnlloVento, 1998). This effect is typically observed for stimuli presented outside the fovea, in the attended contralateral visual field. It is
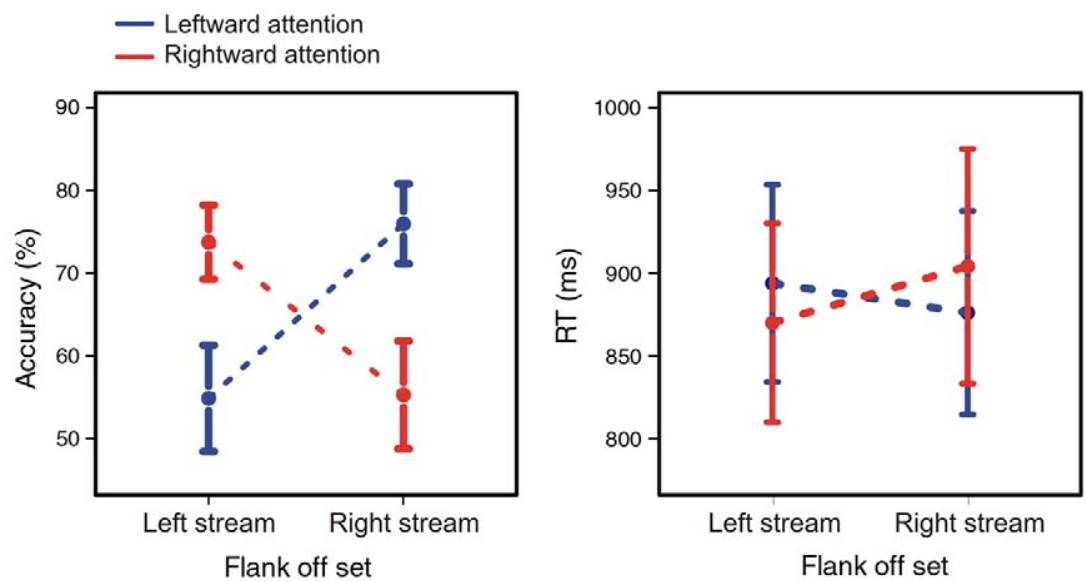

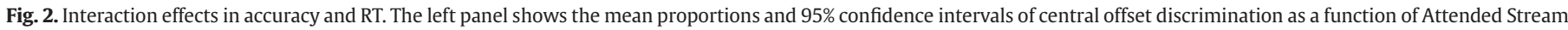

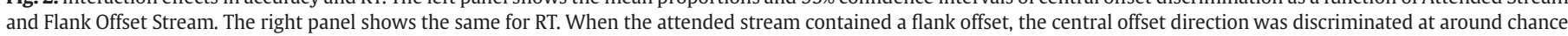
level and RT was increased. When the flank offset was in the unattended stream, however, observers reported the central offset more reliably and quickly. 
a)

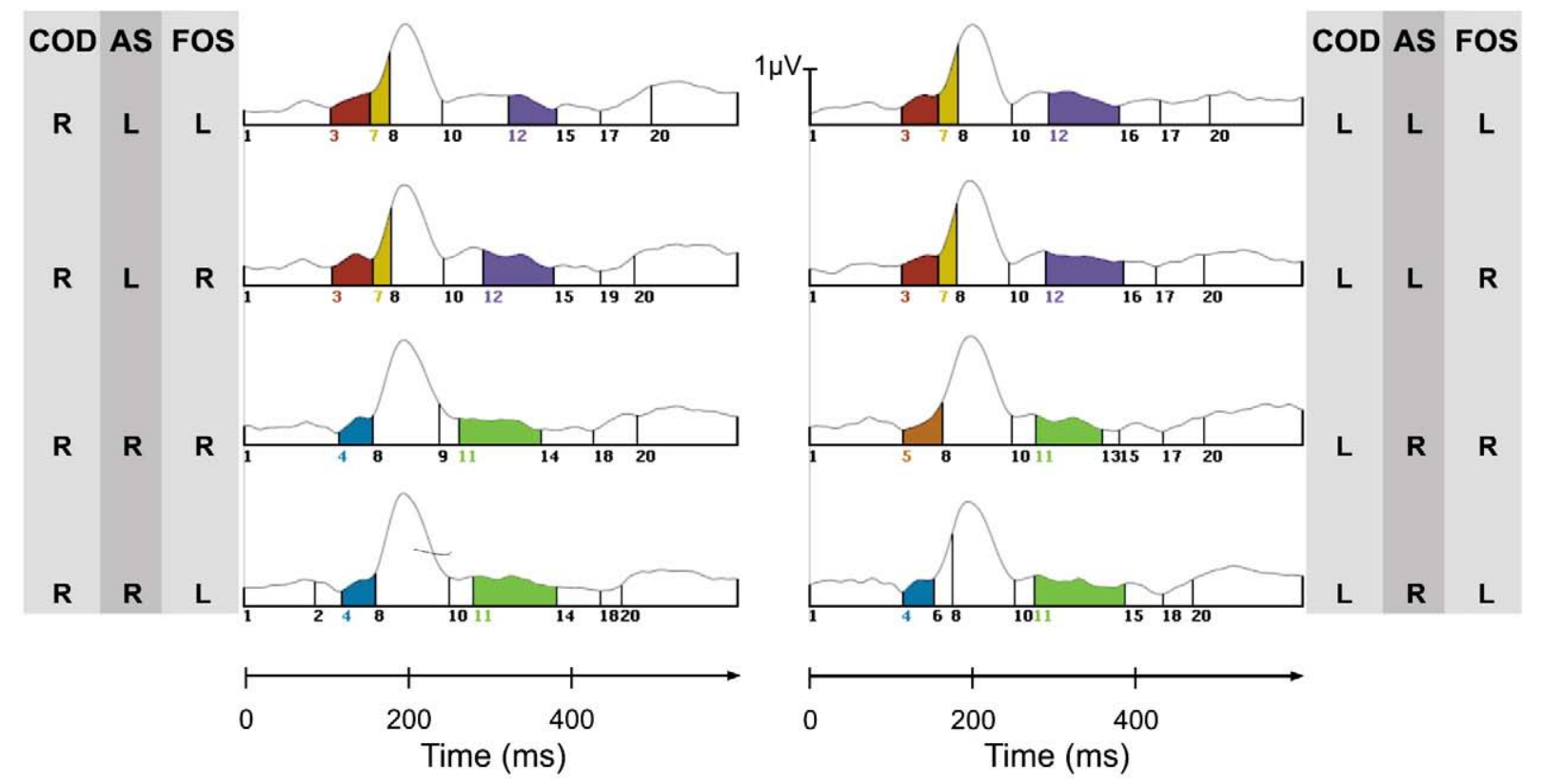

b)
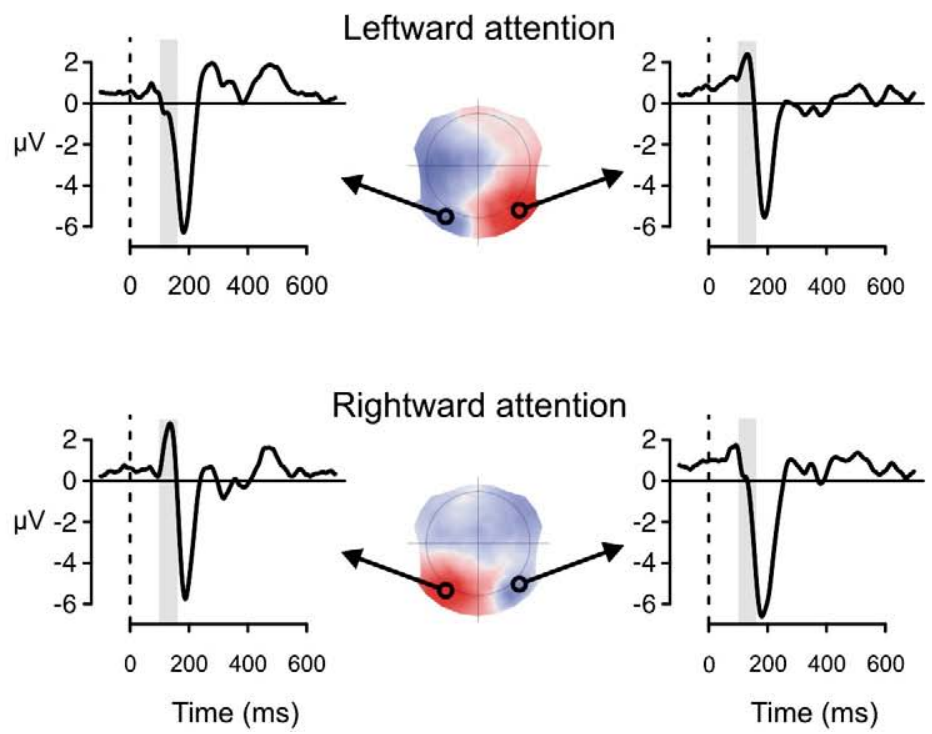

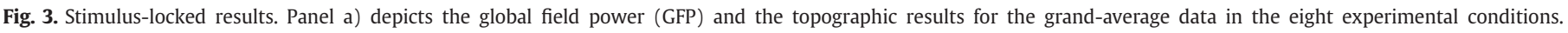

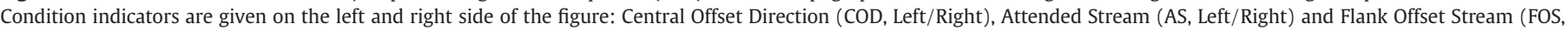

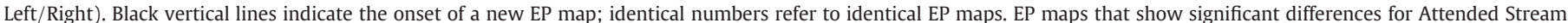

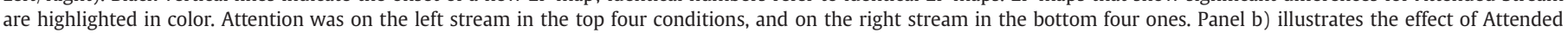

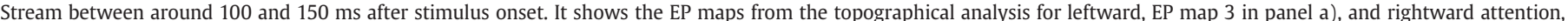

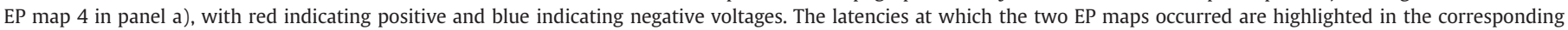

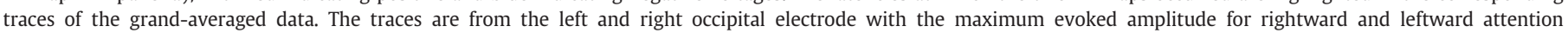
respectively, average referenced.

remarkable that these effects occur for our centrally presented stimuli with an attention shift that spanned less than half a degree of visual angle. Our results suggest that the attended stream is more processed than the unattended stream, in line with the behavioral effects of integration. Non-retinotopic feature integration only occurred within the attended stream. The response-locked data did not reflect whether the attended stream contained a flank offset. That is, they did not reflect non-retinotopic feature integration.

\section{Response-locked analysis}

The topographical analysis of the response-locked EPs showed a variety of EP maps in the period from $-700 \mathrm{~ms}$ to button press, see Fig. 4, that showed no systematic topography differences between conditions. Two EP maps were observed that occurred in all eight conditions. One common EP map immediately preceded the button press (EP map 41). The duration of this map did not change 
a)

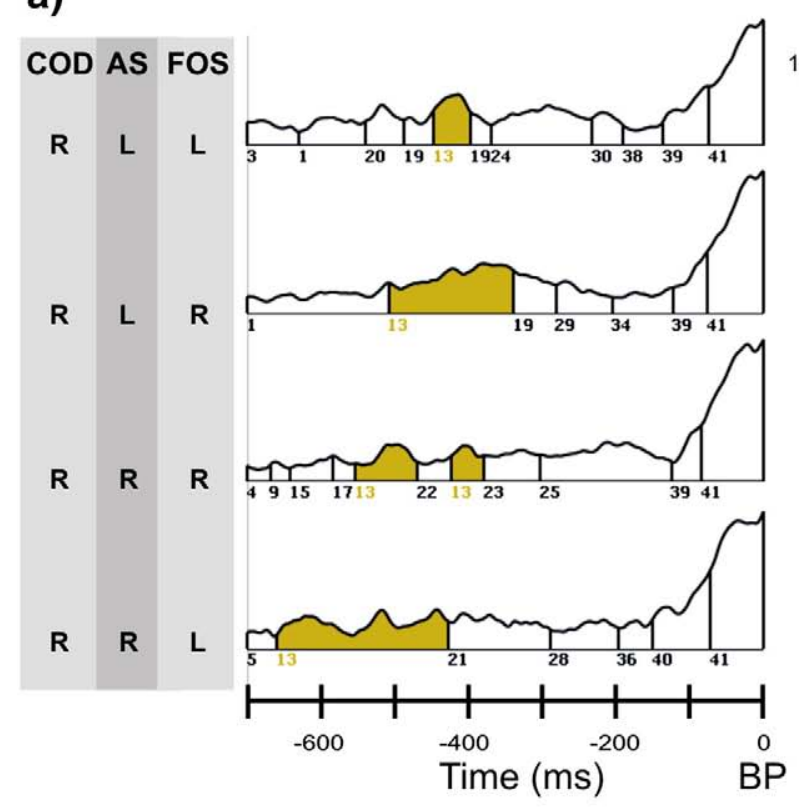

b)

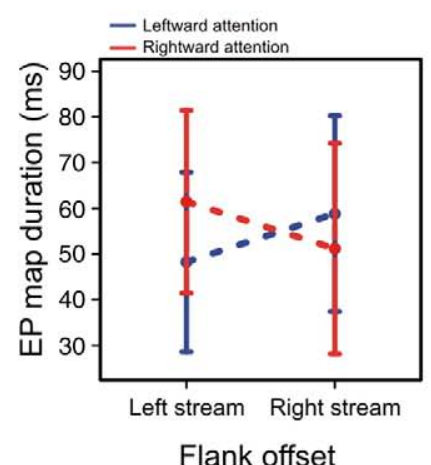

EP map 13

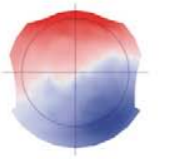

Flank offset

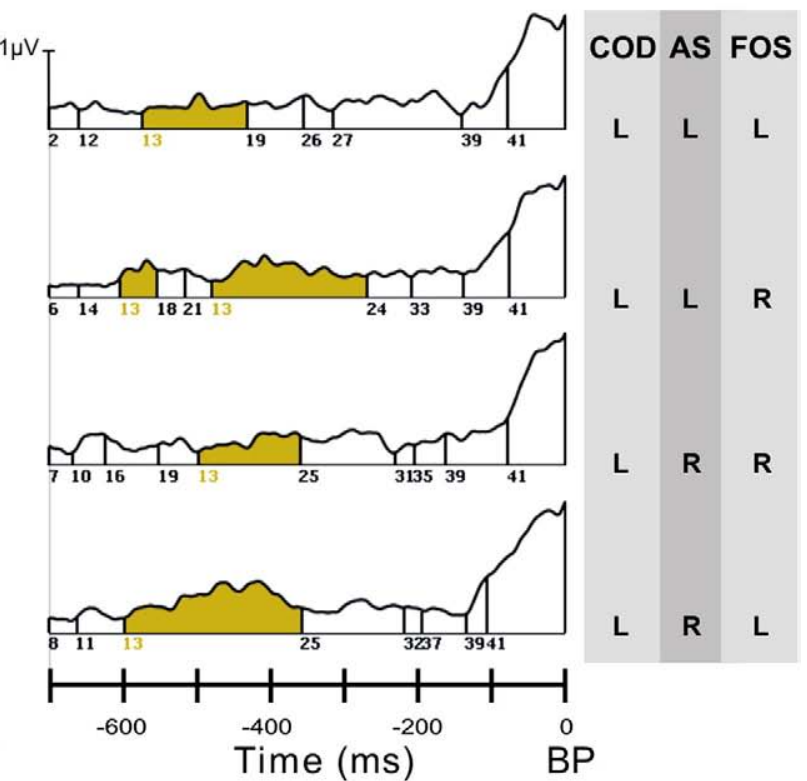

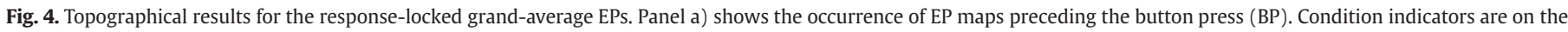

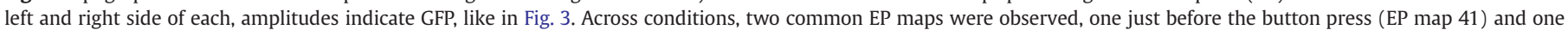

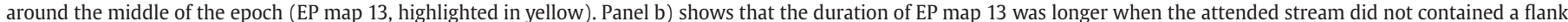
offset (i.e. conditions RLR, RRL, LLR and LRL in panel a) compared to when it contained a flank offset. Error bars denote $95 \%$ confidence intervals.

systematically between conditions and presumably reflects response execution processes. The other common EP map occurred between around $-660 \mathrm{~ms}$ and $-270 \mathrm{~ms}$ to the button press (EP map 13). The duration of this map, as assessed from individual data (see Materials and methods), showed an interaction effect between Attended Stream and Flank Offset Stream, $F_{(1,11)}=6.60, p=0.023$. The map lasted longer when the attended stream did not contain the flank offset, i.e. when the two offsets did not integrate.

Between the first and last occurrence of EP map 13, the time-point by time-point SPM analysis of current densities localized the interaction of Attended Stream and Flank Offset Stream to a network of posterior, frontal, and medial areas. Supplementary Table 1 lists the latencies and spatial locations where significant interaction effects where observed. None of these areas showed a significant three-way interaction, confirming that the reported 2-way interactions can be considered independent from the third factor Central Offset Direction. The SPM results and their time-line are depicted in Fig. 5 (see also Supplementary Movie 2).

For all brain areas showing an interaction effect between Attended Stream and Flank Offset Stream, we obtained a significant correlation between estimated current density and accuracy (see Supplementary Table 1). This correlation was positive, i.e. higher current densities went with higher probabilities of reporting the central offset direction, in all areas except for the Middle Frontal Gyrus. In the Middle Frontal
Gyrus current densities increased when behavior was at chance level, i.e. when the two offsets integrated. Fig. 5 illustrates the observed correlations in the Middle Temporal Gyrus and the Middle Frontal Gyrus.

\section{Discussion}

Using an EP paradigm, we found that neural correlates of nonretinotopic feature integration are time-locked to the response, rather than to stimulus onset. This suggest that endogenously timed neural processes drive feature integration more than those time-locked to the stimulus onset. Using topographical analyses of the EEG, supplemented by SPM of estimated current densities, we show that non-retinotopic feature integration decreases electrical activity in an extensive network of brain areas, including higher-level visual areas, frontal and central ones. The dynamics of the integration effects across these areas showed an orderly progression of differential activity from visual and categorization areas to response-selection and responsepreparation areas.

\section{Stimulus-locked attention effects}

A small, endogenous shift of attention determined whether or not the central offset integrated with the flank offset. It is well known that 

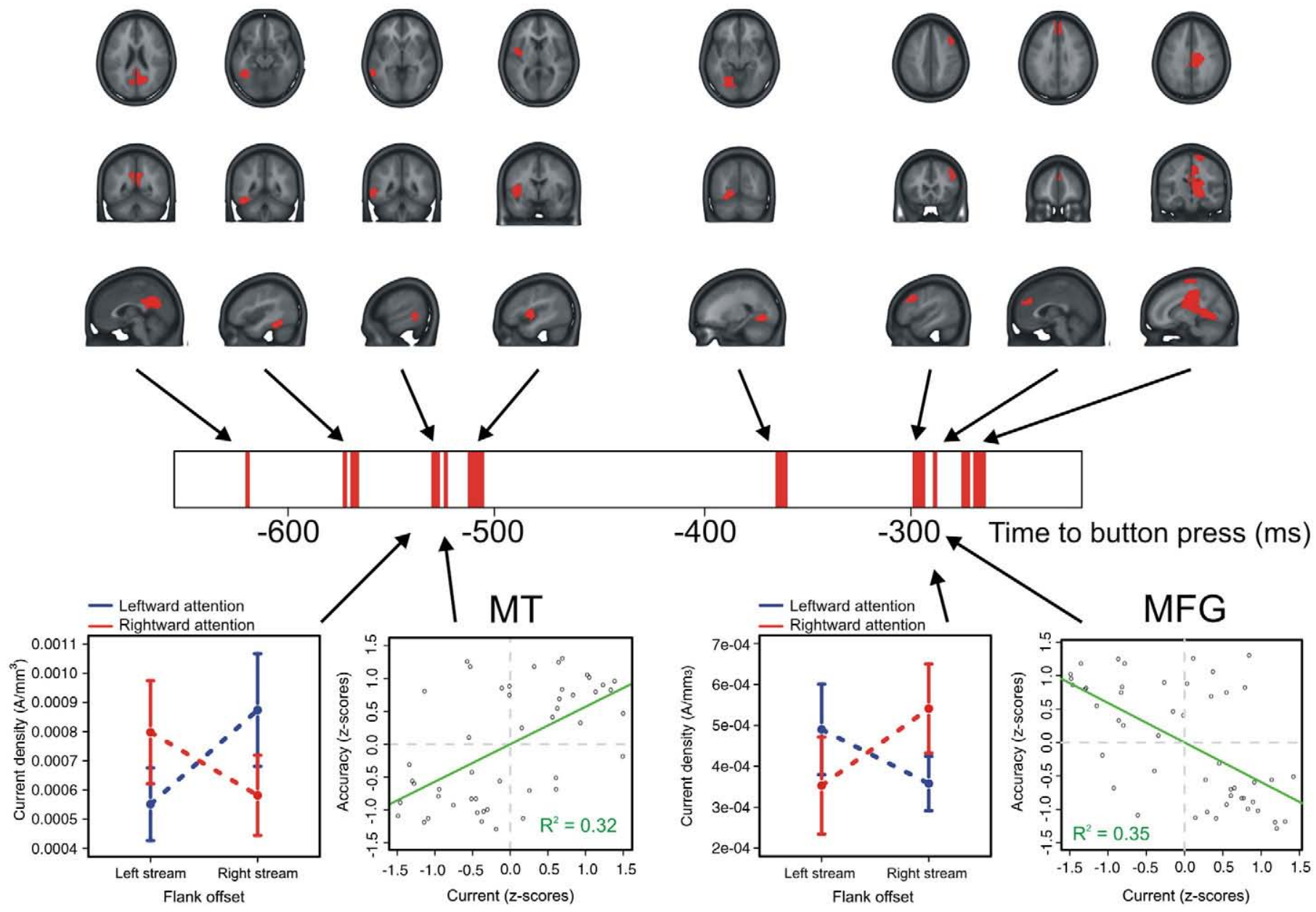

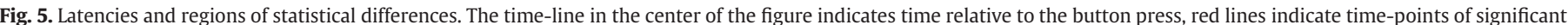

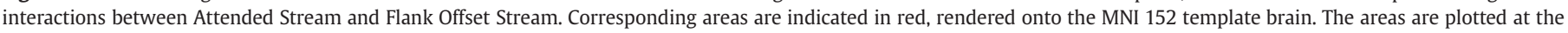

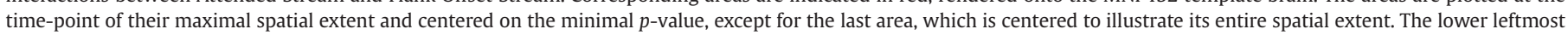

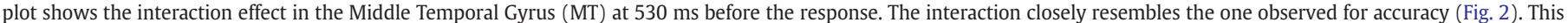

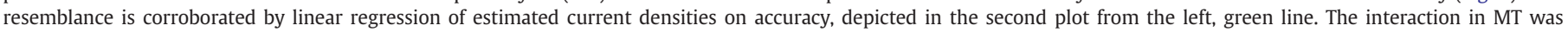

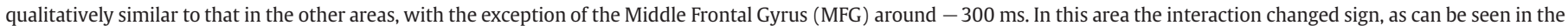
two lower right plots. Here too, a strong correlation with accuracy was observed. In all plots error bars denote 95\% confidence intervals.

selective attention to a peripheral target enhances contralateral EPs from about 100 ms after stimulus onset (Heinze et al., 1990; Hillyard and Anllo-Vento, 1998). We observed similar effects, even though the attention shift consisted of less than half a degree of visual angle (Fig. 3b). Around $100 \mathrm{~ms}$ after stimulus onset the second pair of flankers is on the screen, but only the first flank pair can have evoked activity in visual cortex. The attention effects were therefore evoked by lines separated no more than $400^{\prime \prime}$ ( $1 / 9$ degree).

This shows that small endogenous shifts of attention can have considerable effects on the evoked activity, even when the stimuli are small, highly dynamic, and presented within the fovea. The evoked attention differences, however, do not depend on whether the attended stream contains a flank offset. Interestingly, non-retinotopic feature integration does not seem to have strong stimulus-locked components, it does, however have extensive response-locked components.

\section{A response-locked network}

The correlates of non-retinotopic feature integration were observed together with one stable EP map (Fig. 4). This map may reflect a functional microstate of electrical brain activity that precedes response execution, which typically peaks closer to the button press (Huang et al., 2004). This microstate most likely reflects decisionrelated processes about the experimental task, like discriminating the offset direction in the stream. The state lasts longer when the central offset does not integrate with the flank offset. In that case, visual information is available to categorize the offset (left or right) and select the appropriate response. When the central offset integrates with the flank offset, however, the relevant visual information is presumably unavailable because the two features integrate. The subsequent categorization and response-selection processes then last shorter or can hardly take place, resulting in a shorter microstate. Within the period where this microstate can occur the underlying electrical activity that distinguishes integration from non-integration is highly dynamic and widespread.

Non-retinotopic feature integration is reflected in a network of occipital, frontal, and central areas (Fig. 5). One after another, these regions show decreased activity when the central offset integrates with the flank offset. The decrease first becomes apparent in posterior cortex, including FG and MT. The FG is considered a non-retinotopic area that reflects higher-level object properties. More specifically, the observed decreased activity in left FG suggests an impediment in object categorization processes (Garoff et al., 2005; Koutstaal et al., 2001; Plomp et al., in press). The involvement of MT reflects the motion-dependence of non-retinotopic integration. Subsequently, non-retinotopic integration decreases activity in the left AI, an area involved in perceptual decision making (Heekeren et al., 2004; Pessoa and Padmala, 2005; Preuschoff et al., 2008). The decreased activity is 
in line with previous findings showing that AI activity increases with visual categorization uncertainty (Grinband et al., 2006). When the two offsets integrate, no visual information is available for offsetcategorization, leaving AI activity at baseline. Without integration, however, offset information is available from visual areas. Because the offset size is small and hard to discriminate (individually calibrated to $75 \%$ correct responses), this gives rise to categorization uncertainty, and increased AI activity.

At around $300 \mathrm{~ms}$ before the response, non-retinotopic feature integration decreases activity in the Medial Frontal Gyrus. Although categorization uncertainty is reflected in the Medial Frontal Gyrus, as it is in AI (Grinband et al., 2006), the timing at $-290 \mathrm{~ms}$ may favor an interpretation in terms of response-selection processes (Rushworth et al., 2004). This area possibly reflects the coupling between the categorization (left/right offset) and the response selection (press the left/right button). The subsequent volley of decreased activity in central areas, including the Cingulate Cortex and the Thalamus, may reflect impediments in response selection because these areas are known to be involved in preparing finger movements (Deiber et al., 1996).

The Middle Frontal Gyrus is the only area where electrical brain activity increases with feature integration. Considering its role in monitoring ongoing processes and its sensitivity to response uncertainty (Ridderinkhof et al., 2004), the increase may have been expected. When the two offsets integrate, no visual categorization can take place so that response uncertainty is high and activity in the Middle Frontal Gyrus increases.

Our electrical neuroimaging results are in good agreement with previously observed masking effects. The fMRI studies of Bar et al. (2001) and Green et al. (2005) showed that masking reduces neural activity primarily in LOC and FG. We observed non-retinotopic integration effects in FG but not in LOC. This may be due to the retinotopic organization in lateral occipital areas (Gardner et al., 2008), whereas FG lies outside of retinotopic areas (Halgren et al., 1999). Additional areas that selectively decrease their activity with masking have also been reported. These include occipital areas around the Lingual Gyrus, area MT, the Insula, the Cingulate Cortex, the Middle Frontal and Precentral Gyri as well as the Thalamus (Green et al., 2005; Haynes et al., 2005). Our study shows a notable overlap: all these areas showed selectivity for non-retinotopic integration as determined by SPM of estimated brain activity. While this extends the previous findings to the non-retinotopic domain, our results also show how the differential brain activity progresses from higher-level visual areas, via frontal, to central ones. In addition, we provide evidence that integration related activity is response-locked, rather than stimuluslocked.

Although it cannot be completely excluded that stimulus-locked components contribute to non-retinotopic feature integration, our data suggest that response-locked components are more prominent. Despite the small and highly dynamic stimuli we obtained robust stimulus-evoked responses (Fig. 3b), so that we should have been able, in principle to detect stimulus-locked integration effects. Both the topographical and the SPM analysis showed sensitivity to stimulus-locked differences (see Supplementary Figure 1) even though the critical feature for these effects, the central line offset, was close to the hyperacuity range. In addition, the stimulus-locked analysis covered a long enough period (up to $600 \mathrm{~ms}$ after stimulus onset) to identify effects throughout the hierarchy of visual processing (Lamme and Roelfsema, 2000; Thorpe and Fabre-Thorpe, 2001).

Because our analyses are based on averages across trials, they do not take into account the possibility that induced, as opposed to evoked (Makeig et al., 2002), changes in activity contribute to nonretinotopic feature integration. It could be that oscillatory activity of variable phase (relative to stimulus onset) contributes to nonretinotopic integration. Under that account, ongoing activity at the single trial level sets the pace for integration. Our results are in principle compatible with such an account when we assume that the induced components on a single trial level result in highly synchronized local activity time-locked to the response. Although induced components may play a role, we here focused on the time-locked components of non-retinotopic processing.

Our results show that stimulus-locked components reflect nonretinotopic integration to a much smaller degree than responselocked components do. This suggests that endogenously timed electric brain activity underlies these integration effects. The endogenous timing may be understood from the function of non-retinotopic processes in visual perception. Non-retinotopic processes essentially maintain the identity of perceptual objects across space and time (Otto et al., 2009), that is, they code visual information irrespective of when and where it was presented. With highly dynamic visual information, object identity may be better preserved in a stimulusindependent temporal reference frame. In those cases, part of the evoked brain activity does not tightly follow the timing of the stimulus. This endogenously timed activity then gives rise to a chain of highly synchronized neuronal activity, starting in high-level visual areas, that leads up to the behavioral response.

\section{Methodological considerations}

The spatial accuracy of EEG inverse solutions like LAURA is more limited than that of fMRI. From this it follows that the spatial resolution of statistical images derived from estimated current densities is limited as well, and may be insufficient to differentiate between nearby structures. This concerns in particular the central brain regions, which show greater differentiation per volume, but to a lesser degree also the cortical regions. Our labeling of areas should therefore be taken as gross indicators; additional measurements using the combined spatio-temporal resolution of MEG (Liu et al., 2006; Moradi et al., 2003), or intracranial recordings (Blanke et al., 2005; Blanke et al., 1999) are needed to verify with greater spatial accuracy the brain regions involved in non-retinotopic feature integration.

The limited spatial accuracy of EEG source localizations affects deeper sources more than superficial ones. Deeper sources are most likely underestimated when using a LAURA inverse solution (Grave de Peralta Menendez et al., 2004; Pascual-Marqui, 1999). These limitations can be partially remedied by using a statistical approach (Michel et al., 2004). Even when the estimated activity in deeper brain regions is unrealistically small, statistical analysis can reveal whether differences between experimental conditions are reliable. In this way, for example, differential activity in the thalamus was previously reported in a memory task (Kounios et al., 2001).

The durations of the significant effects within an area were very brief, but may arise from longer lasting underlying differences. The durations of statistical differences cannot be taken to reflect the duration of the differences in individual observers, or single trials. Statistical results only show where and when differences are reliable across observers, they do not indicate that differential brain activity stops and ends there. In fact, individual selectivity around the observed latencies can only last longer than the duration of significant differences across individuals. If individual selectivity lasted shorter, small temporal variations would render them indiscernible across observers. The response-locked network is therefore best viewed as a useful minimal sketch; it shows the most outstanding areas and their timing, but is not an exhaustive description of the presumably more complex neural dynamics underlying non-retinotopic feature integration.

\section{Conclusions}

We used a sequential metacontrast paradigm to induce nonretinotopic feature integration. The results show that stimulus-locked EPs strongly depend on what part of the stimulus is attended, but do 
not reflect non-retinotopic feature integration. These integration processes are reflected more strongly in brain activity time-locked to the behavioral response than in activity time-locked to stimulus onset. Our analysis of source estimates indicates that the responselocked integration effects first decrease activity in higher-level visual areas and subsequently also in categorization and response-selection areas. The activity changes in this network entirely reflect endogenously initiated activation differences for identical stimuli. The endogenous nature of non-retinotopic processes affirms their role of maintaining object identity across space and time.

\section{Acknowledgments}

This work is supported by the Swiss National Science Foundation. The Cartool software (http://brainmapping.unige.ch/Cartool.htm) has been programmed by Denis Brunet, from the Functional Brain Mapping Laboratory, Geneva, Switzerland, and is supported by the Center for Biomedical Imaging (CIBM) of Geneva and Lausanne. We thank Christoph Michel for helpful discussion and Marc Repnow for technical support.

\section{Appendix A. Supplementary data}

Supplementary data associated with this article can be found, in the online version, at doi:10.1016/j.neuroimage.2009.06.031.

\section{References}

Arzy, S., Thut, G., Mohr, C., Michel, C.M., Blanke, O., 2006. Neural basis of embodiment: distinct contributions of temporoparietal junction and extrastriate body area. J Neurosci. 26, 8074-8081.

Aydin, M., Herzog, M.H., Öğmen, H., 2008. Perceived speed differences explain apparent compression in slit viewing. Vision Res. 48, 1603-1612.

Aydin, M., Herzog, M.H., Öğmen, H., 2009. Shape distortions and Gestalt grouping in anorthoscopic perception. J. Vis. 9 (3), 1-8

Bach, M., 1996. The Freiburg Visual Acuity test-automatic measurement of visua acuity. Optom. Vis. Sci. 73, 49-53.

Bachman, T., 1994. Psychophysiology of Visual Masking. Nova Science, Commack, NY.

Bar, M., Tootell, R.B., Schacter, D.L., Greve, D.N., Fischl, B., Mendola, J.D., Rosen, B.R., Dale, A.M., 2001. Cortical mechanisms specific to explicit visual object recognition. Neuron 29, 529-535.

Benjamini, Y., Hochberg, Y., 1995. Controlling the false discovery rate: a practical and powerful approach to multiple testing. J. R. Stat. Soc., Ser. B 57, 289-300.

Blanke, O., Morand, S., Thut, G., Michel, C.M., Spinelli, L., Landis, T., Seeck, M., 1999. Visual activity in the human frontal eye field. Neuroreport 10, 925-930.

Blanke, O., Mohr, C., Michel, C.M., Pascual-Leone, A., Brugger, P., Seeck, M., Landis, T., Thut, G., 2005. Linking out-of-body experience and self processing to mental ownbody imagery at the temporoparietal junction. J. Neurosci. 25, 550-557.

Born, R.T., Bradley, D.C., 2005. Structure and function of visual area MT. Annu. Rev. Neurosci. 28, 157-189.

Breitmeyer, B.G., Öğmen, H., 2006. Visual Masking: Time Slices Through Conscious and Unconscious Vision. Oxford University Press, Oxford.

Breitmeyer, B.G., Herzog, M.H., Öğmen, H., 2008. Motion, not masking, provides the medium for feature attribution. Psychol. Sci. 19, 823-829.

Cavanagh, P., Holcombe, A.O., Chou, W., 2008. Mobile computation: spatiotemporal integration of the properties of objects in motion. J. Vis. 8 (1), 1-23.

Deiber, M.P., Ibanez, V., Sadato, N., Hallett, M., 1996. Cerebral structures participating in motor preparation in humans: a positron emission tomography study. J. Neurophysiol. 75, 233-247.

Engel, S.A., Rumelhart, D.E., Wandell, B.A., Lee, A.T., Glover, G.H., Chichilnisky, E.J., Shadlen, M.N., 1994. fMRI of human visual cortex. Nature 369, 525.

Friston, K.J., Holmes, A.P., Worsley, K.J., Poline, J.B., Frith, C.D., Frackowiak, R.S.J., 1995 Statistical parametric maps in functional imaging: a general linear approach. Hum. Brain Mapp. 2, 189-210.

Gardner, J.L., Merriam, E.P., Movshon, J.A., Heeger, D.J., 2008. Maps of visual space in human occipital cortex are retinotopic, not spatiotopic. J. Neurosci. 28, 3988-3999.

Garoff, R.J., Slotnick, S.D., Schacter, D.L., 2005. The neural origins of specific and genera memory: the role of the fusiform cortex. Neuropsychologia 43, 847-859.

Genovese, C.R., Lazar, N.A., Nichols, T., 2002. Thresholding of statistical maps in functional neuroimaging using the false discovery rate. Neurolmage 15, 870-878.

Grave de Peralta Menendez, R., Murray, M.M., Michel, C.M., Martuzzi, R., Gonzalez Andino, S.L., 2004. Electrical neuroimaging based on biophysical constraints. Neurolmage 21, 527-539.

Green, M.F., Glahn, D., Engel, S.A., Nuechterlein, K.H., Sabb, F., Strojwas, M., Cohen, M.S 2005. Regional brain activity associated with visual backward masking. J. Cogn. Neurosci. 17, 13-23.

Grill-Spector, K., Kourtzi, Z., Kanwisher, N., 2001. The lateral occipital complex and it role in object recognition. Vision Res. 41, 1409-1422.
Grinband, J., Hirsch, J., Ferrera, V.P., 2006. A neural representation of categorization uncertainty in the human brain. Neuron 49, 757-763.

Halgren, E., Dale, A.M., Sereno, M.I., Tootell, R.B., Marinkovic, K., Rosen, B.R., 1999. Location of human face-selective cortex with respect to retinotopic areas. Hum. Brain Mapp. 7, 29-37.

Haynes, J.D., Driver, J., Rees, G., 2005. Visibility reflects dynamic changes of effective connectivity between V1 and fusiform cortex. Neuron 46, 811-821.

Heekeren, H.R., Marrett, S., Bandettini, P.A., Ungerleider, L.G., 2004. A general mechanism for perceptual decision-making in the human brain. Nature 431, 859-862.

Heinze, H.J., Luck, S.J., Mangun, G.R., Hillyard, S.A., 1990. Visual event-related potentials index focused attention within bilateral stimulus arrays. I. Evidence for early selection. Electroencephalogr. Clin. Neurophysiol. 75, 511-527.

Herzog, M.H., Koch, C., 2001. Seeing properties of an invisible object: feature inheritance and shine-through. Proc. Natl. Acad. Sci. U. S. A. 98, 4271-4275.

Hillyard, S.A., Anllo-Vento, L., 1998. Event-related brain potentials in the study of visual selective attention. Proc. Natl. Acad. Sci. U. S. A. 95, 781-787.

Huang, M.X., Harrington, D.L., Paulson, K.M., Weisend, M.P., Lee, R.R., 2004. Temporal dynamics of ipsilateral and contralateral motor activity during voluntary finger movement. Hum. Brain Mapp. 23, 26-39.

Kastner, S., Pinsk, M.A., De Weerd, P., Desimone, R., Ungerleider, L.G., 1999. Increased activity in human visual cortex during directed attention in the absence of visual stimulation. Neuron 22, 751-761.

Kawabe, T., 2008. Spatiotemporal feature attribution for the perception of visual size. J. Vis. 8 (7), 1-9.

Kounios, J., Smith, R.W., Yang, W., Bachman, P., D'Esposito, M., 2001. Cognitive association formation in human memory revealed by spatiotemporal brain imaging. Neuron 29, 297-306.

Koutstaal, W., Wagner, A.D., Rotte, M., Maril, A., Buckner, R.L., Schacter, D.L., 2001. Perceptual specificity in visual object priming: functional magnetic resonance imaging evidence for a laterality difference in fusiform cortex. Neuropsychologia 39, 184-199.

Lamme, V.A., Roelfsema, P.R., 2000. The distinct modes of vision offered by feedforward and recurrent processing. Trends Neurosci. 23, 571-579.

Lehmann, D., 1984. EEG assessment of brain activity: spatial aspects, segmentation and imaging. Int. J. Psychophysiology 1, 267-276.

Lehmann, D., Skrandies, W., 1980. Reference-free identification of components of checkerboard-evoked multichannel potential fields. Electroencephalogr. Clin. Neurophysiol. 48, 609-621.

Lehmann, D., Ozaki, H., Pal, I., 1987. EEG alpha map series: brain micro-states by spaceoriented adaptive segmentation. Electroencephalogr. Clin. Neurophysiol. 67 (3), 271-288.

Lehmann, D., Strik, W.K., Henggeler, B., Koenig, T., Koukkou, M., 1998. Brain electric microstates and momentary conscious mind states as building blocks of spontaneous thinking: I. Visual imagery and abstract thoughts. Int. J. Psychophysiology 29,1-11.

Liu, L., Plomp, G., van Leeuwen, C., Ioannides, A.A., 2006. Neural correlates of priming on occluded figure interpretation in human fusiform cortex. Neuroscience 141,1585-1597.

Makeig, S., Westerfield, M., Jung, T.P., Enghoff, S., Townsend, J., Courchesne, E., Sejnowski, T.J., 2002. Dynamic brain sources of visual evoked responses. Science 295, 690-694.

Mercier, M., Schwartz, S., Michel, C.M., Blanke, O., 2009. Motion direction tuning in human visual cortex. Eur. J. Neurosci. 29, 424-434.

Michel, CM, Thut, G, Morand, S, Khateb, A, Pegna, AJ, Grave de Peralta, R, Gonzalez, S, Seeck, M, Landis, T., 2001. Electric source imaging of human brain functions. Brain Res. Brain Res. Rev. 36 (2-3), 108-118.

Michel, C.M., Murray, M.M., Lantz, G., Gonzalez, S., Spinelli, L., Grave de Peralta, R., 2004. EEG source imaging. Clin. Neurophysiol. 115, 2195-2222.

Moradi, F., Liu, L.C., Cheng, K., Waggoner, R.A., Tanaka, K., Ioannides, A.A., 2003. Consistent and precise localization of brain activity in human primary visual cortex by MEG and fMRI. NeuroImage 18, 595-609.

Murray, MM, Molholm, S, Michel, CM, Heslenfeld, DJ, Ritter, W, Javitt, DC, Schroeder, CE, Foxe, JJ., 2005. Grabbing your ear: rapid auditory-somatosensory multisensory interactions in low-level sensory cortices are not constrained by stimulus alignment. Cereb. Cortex 15 (7), 963-974.

Murray, M.M., Brunet, D., Michel, C.M., 2008. Topographic ERP analyses: a step-by-step tutorial review. Brain Topogr. 20, 249-264.

Nishida, S., Watanabe, J., Kuriki, I., Tokimoto, T., 2007. Human visual system integrates color signals along a motion trajectory. Curr. Biol. 17, 366-372.

Öğmen, H., Otto, T.U., Herzog, M.H., 2006. Perceptual grouping induces non-retinotopic feature attribution in human vision. Vision Res. 46, 3234-3242.

Oldfield, R.C., 1971. The assessment and analysis of handedness: the Edinburgh inventory. Neuropsychologia 9, 97-113.

Otto, T.U., Öğmen, H., Herzog, M.H., 2006. The flight path of the phoenix-the visible trace of invisible elements in human vision. J. Vis. 6, 1079-1086.

Otto, T.U., Öğmen, H., Herzog, M.H., 2009. Feature integration across space, time, and orientation. J. Exp. Psychol. Hum. Percept. Perform. doi:10.1037/a0015798.

Pascual-Marqui, R.D., 1999. Review of methods for solving the EEG inverse problem. Int. J. Bioelectromagn. 1, 75-86.

Pascual-Marqui, R.D., Michel, C.M., Lehmann, D., 1995. Segmentation of brain electrical activity into microstates: model estimation and validation. IEEE Trans. Biomed. Eng. 42, 658-665.

Perrin, F., Pernier, J., Bertrand, O., Giard, M.H., Echallier, J.F., 1987. Mapping of scalp potentials by surface spline interpolation. Electroencephalogr. Clin. Neurophysiol. 66, 75-81.

Pessoa, L., Padmala, S., 2005. Quantitative prediction of perceptual decisions during near-threshold fear detection. Proc. Natl. Acad. Sci. U. S. A. 102, 5612-5617.

Pinheiro, J.C., Bates, D.M., 2000. Mixed-Effects Models in S and S-PLUS. Springer 
Plomp, G., van Leeuwen, C., Ioannides, A.A., in press. Functional Specialization and Dynamic Resource Allocation in Visual Cortex. Hum. Brain Mapp. doi:10.1002/hbm.20840.

Preuschoff, K., Quartz, S.R., Bossaerts, P., 2008. Human insula activation reflects risk prediction errors as well as risk. J. Neurosci. 28, 2745-2752.

Ridderinkhof, K.R., Ullsperger, M., Crone, E.A., Nieuwenhuis, S., 2004. The role of the medial frontal cortex in cognitive control. Science 306, 443-447.

Rushworth, M.F., Walton, M.E., Kennerley, S.W., Bannerman, D.M., 2004. Action sets and decisions in the medial frontal cortex. Trends. Cogn. Sci. 8, 410-417.

Sereno, M.I., Pitzalis, S., Martinez, A., 2001. Mapping of contralateral space in retinotopic coordinates by a parietal cortical area in humans. Science 294, 1350-1354.

Shimozaki, S.S., Eckstein, M., Thomas, J.P., 1999. The maintenance of apparent luminance of an object. J. Exp. Psychol. Hum. Percept. Perform. 25, 1433-1453.

Spinelli, L., Andino, S.G., Lantz, G., Seeck, M., Michel, C.M., 2000. Electromagnetic inverse solutions in anatomically constrained spherical head models. Brain Topogr. 13 115-125.

Talairach, J., Tournoux, P., 1988. Co-Planar Stereotaxic Atlas of the Human Brain. G. Thieme, Stuttgart.

Taylor, M.M., Creelman, C.D., 1967. PEST: efficient estimates on probability functions.
J. Acoust. Soc. Am. 41, 782-787.

Thierry, G, Martin, CD, Downing, P, Pegna, AJ., 2007. Controlling for interstimulus perceptual variance abolishes N170 face selectivity. Nat. Neurosci. 10 (4), 505-511.

Thorpe, S.J., Fabre-Thorpe, M., 2001. Neuroscience. Seeking categories in the brain. Science 291, 260-263.

Tootell, R.B., Reppas, J.B., Kwong, K.K., Malach, R., Born, R.T., Brady, T.J., Rosen, B.R., Belliveau, J.W., 1995. Functional analysis of human MT and related visual cortical areas using magnetic resonance imaging. J. Neurosci. 15, 3215-3230.

Tootell, R.B., Hadjikhani, N.K., Vanduffel, W., Liu, A.K., Mendola, J.D., Sereno, M.I., Dale, A M., 1998. Functional analysis of primary visual cortex (V1) in humans. Proc. Natl. Acad. Sci. U. S. A. 95, 811-817.

Watanabe, J., Nishida, S., 2007. Veridical perception of moving colors by trajectory integration of input signals. Journal of Vision 7(11):3, 1-16.

Worsley, K.J., Evans, A.C., Marrett, S., Neelin, P., 1992. A three-dimensional statistical analysis for CBF activation studies in human brain. J. Cereb. Blood Flow Metab. 12, 900-918.

R-Development-Core-Team, 2008. R: A language and environment for statistical computing. 